\title{
An Improved PSO Algorithm Based CommunityTopic Refinement Strategy for Social Network
}

\author{
Lin Cui ${ }^{1,2}$, Caiyin Wang ${ }^{1}$ and Xiaoyin $\mathrm{Wu}^{1}$ \\ ${ }^{1}$ Intelligent Information Processing Laboratory, Suzhou University, Suzhou \\ 234000, Anhui, China \\ ${ }^{2}$ College of Computer Science and Technology, Nanjing University of Aeronautics \\ and Astronautics, Nanjing \\ 210016, Jiangsu, China \\ jsjxcuilin@nuaa.edu.cn, caiyinwang@163.com,xiaoyinwu@126.com
}

\begin{abstract}
Aiming at the division roughness of topic classification existing in the most online social networks community, the improved particle swarm optimization algorithm is applied to refine community topics and concepts of community seeds and community topic are also introduced. In this paper, first of all, the explicit links existing in the community are mined, and the basic community structure is constructed, then the community content is deeply analyzed, according to implicit feature between nodes under online community, community topic categories are elaborately refined until structure is stable. Experiments show that this proposed algorithm can accelerate the convergence of the node and greatly improves the topic mining accuracy of online social network compared with the state-ofart CR2NDAS model and PLSA model.
\end{abstract}

Keywords: Improved particle swarm optimization algorithm, online social network, Community topic, Community seed, Topic refinement

\section{Introduction}

In recent years, with the rapid development of Web 2.0 technology, online social network and instant information system rapidly become popular, which have an increasingly important impact on people. Social network analysis presents a new research hotspot. Social network is abstracted as a graph, in which, the vertices represent persons in online social network, and edges denote relationship between verticles. Among research hotspots on online social network, the community structure has become one of hot issues of social network research [1].

Under online social network, users can create groups or communities according to different topics, they can also join the communities or groups created by others for discussion, obtaining information and communicating with each other [2].With the rapid growth of the number of users under online social networks, the amount of online group or community has increased dramatically; it is more and more difficult for the user to find the community to obtain the really interesting content from such a huge network [3].

For example, on the Internet, a lot of knowledge sharing and exchange activities are published every day, among which, questions and answers community (Q\&A community) provides a network platform for users to ask questions and answer questions. Commonly used answers community are Yahoo Answers, Baidu Zhidao and Sina iask. In these Q\&A communities, users put forward many problems every day, in addition, some other users generally browse the questions they are interested and answer them. In order to answer the questions as soon as possible, it is necessary to carry out a detailed classification for the questions; the questions of similar content are divided into a class. In this way, the 
respondents will find out the interesting problems, and then answer these questions they are very interested in, so that the problem can be solved in time.

The improved particle swarm optimization algorithm which is abbreviated as PSO algorithm, introducing the concept of community seed and the community topic. This paper first analyzes the explicit links existing between community nodes, builds the basic community structure of nodes; then, deeply analyzes the community topic content, defines the community topic and community seed on the basis of the implicit behavior feature between nodes, elaborately refines the communities until the community topic structure is stable.

The remainder of the paper is organized as follows. In the next section, some related works are introduced in detail. In Section 3, PSO algorithm is illustrated and its details are also given out. Section 4 describes our proposed community topic refinement strategy based on PSO algorithm. Section 5 elaborates our experiments on two real-world data sets from DBLP and Baidu Zhidao, and compares our proposed method with CR2NDAS model and PLSA model. Finally, Section 6 summarizes the whole paper briefly and points out directions for future works.

\section{Related Work}

Nowadays, community recommendation and community identification are both important research areas of social network analysis. There are some following books and literatures related to these two researches.

About the community recommendation, Literature [4] proposed decision tree analysis algorithm based on the network topology structure, analyzed network topology structure, analyzed various attributes of individual and direct connection between individuals through the link between individuals and predicted which community individual would join through the method of decision tree. Literature [5] proposed a community recommendation algorithm based on group similarity, other communities were recommended for the members of the community by calculating the similarity between groups. Literature [6] performed community recommendation by group clustering method of the social network.

On community identification, literature [7] constructed a social spending model to find community structure dynamic network. Literature [8] proposed the incremental algorithm to identify the dynamic community, but this method only applies to unchanged network community. Literature [9] adopted GN algorithm to divide each snapshot, the communities were grouped into the community graph, once again using GN algorithm to partition the graph and get the dynamic community. Literature [10] proposed a dynamic adaptive algorithm using Newman algorithm to update the community structure module. Literature [11] proposed a dynamic social network community discovery algorithm based on the random walk theory, but the time complexity was not improved; in order to reduce time overhead, partitioning algorithm based on incremental mode has been widely concerned. Literature [12] proposed an adaptive dynamic community.

Although a lot of researches have been done on the community research, there are some problems that still cannot been solved, such as most research algorithms on online community only surround the static characteristics of the community, such as link analysis is mainly on the static data analysis or according to dynamic data and adopting statistical method to obtain the analysis of static network diagram, prediction and recommendation accuracy rate are very low, and this method cannot analyze the changes in the influence of individual effect on the social network and network evolution. There is no detailed and in-depth analysis of the community topic characteristics. Aiming at these reasons, improved particle swarm optimization algorithm is introduced to elaborately refine topic community until the topic structure of community is stable. 


\section{Introduction of PSO Algorithm}

Particle Swarm Optimization (PSO) algorithm is an evolutionary algorithm based on swarm intelligence approach proposed by Kennedy and Eberhart in 1995 [13]. In PSO algorithm, the feasible solution of each optimization problem is a particle in space. Assuming there $N$ particles movement at $D$ dimensional space, $v_{i}=\left(v_{i, 1}, v_{i, 2} \cdots v_{i, D}\right)$ denotes velocity vector of particle $i, X_{i}=\left(x_{i, 1}, x_{i, 2} \cdots x_{i, D}\right)$ is position vector space of particle $i$. According to $v_{i}$ and $X_{i}$, particle $i$ continuously adjusts its velocity and space position, until it reaches the global optimal solution. The optimal solution of the particle itself is represented by $P_{b e s t}, P_{b e s t}=\left(p_{i, 1}, p_{i, 2} \cdots p_{i, D}\right)$, the optimal solution that population found in the current time is denoted by $g_{\text {best }}$, $g_{\text {best }}=\left(g_{i, 1}, g_{i, 2} \cdots g_{i, D}\right) \cdot v_{i}{ }^{t}{ }^{t}=\left(v_{i, 1}{ }^{t}, v_{i, 2}{ }^{t} \cdots v_{i, D}{ }^{t}\right)$ is the iteration speed of particle $i$ in the $i$ times, so the speed of iteration in the $t+1$ times is :

$$
v_{i}^{(t+1)}=w v_{i}^{t}+c_{1} \operatorname{rand}()\left(P_{b i}-X_{i}^{(t)}\right)+c_{2} \operatorname{rand}()\left(g_{b i}-X_{i}^{(t)}\right)
$$

In the formula (1), w is called the inertia factor and is greater than $0, c_{1}$ is a cognitive learning factor, $c_{2}$ is a social learning factors, $c_{1}$ and $c_{2}$ are positive constants, $\operatorname{rand}()$ is a function that can produce random number in the range of $[0,1]$. The particle velocity value of each dimension is in $\left[v_{\min }, v_{\max }\right]$, if the velocity of the particle in somedimensional space is not in the range of $\left[v_{\min }, v_{\max }\right]$, the current speed of the particle would be set the corresponding threshold. When iterating in $t+1$ times, the position of particle $i$ is shown in the following formula (2):

$$
X_{i}^{(t+1)}=X_{i}^{(t)}+V_{i}^{(t+1)}
$$

When actually applying PSO algorithm, if the number of iterations reach the maximum or particles finally find the optimal position that meets the minimum threshold, iteration is terminated [14].Applying PSO algorithm to research community topic refinement strategy, each node in the community is regarded as a particle in the particle swarm, the particles in the community continuously exchange and share information, the particles with similar or identical contents are been polymerization to the same sub topic categories, forming various sub topic community, so that topic correlation among similar nodes in the same sub topic community is higher than the topic correlation among nodes in different sub topics community.

\section{The Proposed Community Topic Refinement Strategy Based on PSO Algorithm}

Under the online community, each node is regarded as a particle, particles depend on their personal properties, constantly seek and explore the most suitable location and finally establish contact with nodes that have the same or similar topic contents. Particles work together to build a stable sub community, which can provide a good reference information for the related topic community. The detailed steps of community topic refinement strategy based on PSO algorithm are as follows:

(1) According to the direct links between nodes, the explicit structure between nodes is mined. In the online community, the closer the connections between nodes are, the more proof that these nodes belong to a sub topic community. According to the centrality of nodes selected from the sub topic community, each sub topic community seed is the center particle of this sub topic community. To determine the sub topic community that 
some node $i$ belongs to, we adopt the formula (3), among which, $\{Q A\}$ represents a collection of sub topic community nodes.

$$
R_{Q A}{ }^{i}=\frac{\sum_{j \in\{Q A\}}\left[E_{i, j} \times d_{j}\right]}{\sum_{k \in\{Q A\}}\left[E_{i, k} \times d_{k}\right]}
$$

In the formula (3), if there exists correlation between node $i$ and node $j, E_{i, j}=1$, if there is no correlation between node $i$ and node $j, E_{i, j}=0$. Variable $d_{j}$ denotes correlation degree of node $j$, the bigger $d_{j}$, the closer node $j$ is at the core position. When the correlation degree with the core node is larger, the core nodes which the sub topic of community ties is closer. Givien $\xi$ is the minimum correlation threshold, if $R_{Q A}^{i}>\xi$, the node $i$ belongs to the sub community $Q A$, otherwise, node $i$ is the new sub community seed. If the correlation degrees between and sun topic community $Q A$ and $Q B$ satisfy $R_{Q A}>\xi \& \& R_{Q B}>\xi$, and $\left|R_{Q A}-R_{Q B}\right|<\varepsilon i$, then node $i$ both belong to the sub topic community $Q A$ and $Q B$, so node $i$ is called edge fuzzy particles and needs to be focused on dynamic tracking.

From the above introduction, it can be found out that when mining explicit link between nodes, only the static relationship between nodes is considered, influence of behavior characteristics and content features of user nodes on the community topic is ignored, the divided sub topic community is still rough. For further fine division of the community topic, based on mining community nodes implicit relation, the content characteristic and user behavior features of nodes are analyzed in detail, the community topic is further elaborately divided. Further refinement of sub community algorithm is as follows:

(2) Dig the content features of particles and further divide the community topic in detail. Suppose the number of community is $N$, through mining the explicit structure, $M$ sub topic communities are formed. In the feature vector collection of each sub topic community, the top-k keywords are selected out according to the highest appearance frequency as topic features of the sub community which is denoted by theme $Q A^{\prime}$, themeQA' $=\left(\right.$ keyword $_{1}$, keyword $_{2} \cdots$ keyword $\left._{K}\right)$ represents the number of keywords appearing in the sub topic community. And fitness function that node $i$ is in the sub community $Q A^{\prime}$ is:

$$
A F\left(i, Q A^{\prime}\right)=\frac{\left.\sum_{j=1}^{K} \text { (keyword }_{j} \times X_{Q A^{\prime}, j} \times x_{i, j}\right)}{\sqrt{\sum_{t=1}^{K} X_{Q A^{\prime}, k}^{2}}}
$$

In the formula (4), if keyword ${ }_{j}$ appear in the feature vector of node $i$ and sub community features theme themeQA' of node $i$ are the same, then keyword $_{j}=1$, and

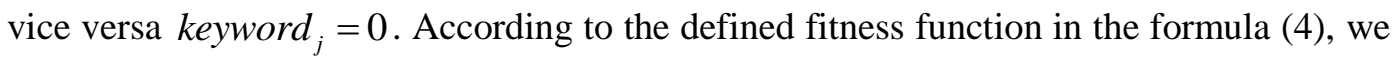
calculate fitness value $A F\left(i, Q A^{\prime}\right)$ between node $i$ and the sub community $Q A^{\prime}$. Set $\lambda$ is the minimum fitness threshold, if $A F\left(i, Q A^{\prime}\right)>\lambda$, node $i$ will remain on the sub node community; if $A F\left(i, Q A^{\prime}\right)<\lambda$, it shows that contents property features and the topic 
features of the sub community $Q A^{\prime}$ do not adapt, node $i$ will be out of t sub topic community $Q A^{\prime}$, then a new node $i$ is regarded as a new community seed, or join a sub community in which its content is similar with node $i$. In addition, as can be seen from the formula that computes $A F\left(i, Q A^{\prime}\right)$, the greater $A F\left(i, Q A^{\prime}\right)$ value is, the greater the similarity between node $i$ and the sub topic of the community $Q A^{\prime}$ is. In the same sub community, because there are the same or similar attributes feature between particles, interaction and sharing information between these particles are more frequent.

(3) Dig the behavior characteristics of user nodes, update and compute feature vector of the user nodes to further update the position where the user is in the sub community according to the behavior feature changes and the change of topics in the sub community. On the basis of the algorithm steps (2), suppose in the D-dimensional user interest space, in the time of $t$, the velocity of nodes is denoted by $v_{t i}=\left(v_{t i, 1}, v_{t i, 2} \cdots v_{t i, D}\right), v_{t i}$ also represents the change degree of user interests in the cycle of $t$; user $_{i}=\left(\right.$ keyword $_{i, 1}$, keyword $_{i, 2} \cdots$ keyword $\left._{i, D}\right)$ is the feature vectors of the node user $i$, keyword $_{i, D}$ represents feature keywords collection that node user $i$ is in $D$ dimensional interest space. According to the change of the user behavior feature and the change of user interesting topic, the position of user topics is updated, which is shown in the formula (5):

$$
\operatorname{user}_{i}^{t+1}=\operatorname{user}_{i}^{t}+v_{i}^{t+1}
$$

(4) If the fitness values of particles in each sub community is greater than the minimum fitness threshold $\lambda$, and the node user finally finds the most suitable sub community location with the changes of interesting topic, fine division of the community topic will reach a steady state, the cycle is finished. Otherwise go to step (2).

\section{Experimental Results and Analysis}

\subsection{Datasets and Setting}

In order to test the proposed community topic refinement strategy based on PSO algorithm, we adopt two real world datasets for experimental analysis and comparison, which are from DBLP and Baidu Zhidao respectively.

(1) Dataset from DBLP database

The first data source we choose is DBLP (http://dblp.uni-trier.de/).DBLP is a wellknown dataset website including a very large number of conferences and papers in computer science,which is an online social network that is composed of the papers' cooperation relationship.In DBLP, communities and friends relationship are all clearly defined,all authors of the same paper is friends and the same category conference all belong to one community. Thus making it an ideal source for our experiments. Using web spider, the needed DBLP dataset is obtained. We maily grabbed the number of 71,069 DBLP data which are from three categories that are artificial intelligence, hardware/architecture and theory/methods. The training set is shown in Table 1, then we randomly extract 3,000 papers from each sub training set without replacement and construct the testing set. 
Table 1. Division of Experimental Dataset from DBLP

\begin{tabular}{|c|r|c|c|}
\hline \multicolumn{2}{|c|}{ Training set } & \multicolumn{2}{c|}{ Testing set } \\
\hline Category & $\begin{array}{c}\text { Number } \\
\text { of Papers }\end{array}$ & Category & $\begin{array}{c}\text { Number } \\
\text { of Papers }\end{array}$ \\
\hline Artificial Intelligence & 32,176 & Artificial Intelligence & 3000 \\
\hline $\begin{array}{c}\text { Hardware/Architectur } \\
\text { e }\end{array}$ & 23,251 & Hardware/Architecture & 3000 \\
\hline Theory/Methods & 15,642 & Theory/Methods & 3000 \\
\hline Total & $\mathbf{7 1 , 0 6 9}$ & Total & $\mathbf{9 0 0 0}$ \\
\hline
\end{tabular}

(2) Dataset from Baidu Zhidao database

The second dataset we employ for evaluation is dataset from Baidu Zhidao database (http://zhidao.baidu.com/), which is the largest Chinese interactive Q\&A platform in the world and also is a typical online social network.Using web spider, Q\&A dataset is grabbed and construct our used experimental dataset. We maily grabbed the number of 104,899 Q\&A data as training set which are about three categories that are computer/network, business/finance and entertainment/leisure respectively. Then 3000 Q\&A nodes from each sub testing set are randomly extracted without replacement and construct a whole testing set. The statistics of the second dataset is shown in Table 2:

Table 2. Division of Experimental Dataset from Baidu Zhidao

\begin{tabular}{|c|c|c|c|}
\hline \multicolumn{2}{|c|}{ Training Set } & \multicolumn{2}{c|}{ Testing Set } \\
\hline Category & $\begin{array}{c}\text { Number } \\
\text { of Q\&A } \\
\text { Pairs }\end{array}$ & Category & $\begin{array}{c}\text { Number } \\
\text { of Q\&A } \\
\text { Pairs }\end{array}$ \\
\hline Computer/Network & 28,837 & Computer/Network & 3000 \\
\hline Business/Finance & 40,423 & Business/Finance & 3000 \\
\hline Entertainment/Leisure & 35,639 & $\begin{array}{c}\text { Entertainment/Leisu } \\
\text { re }\end{array}$ & 3000 \\
\hline Total & $\mathbf{1 0 4 , 8 9 9}$ & Total & $\mathbf{9 0 0 0}$ \\
\hline
\end{tabular}

Our experiments are conducted on a personal computer equipped with an Intel Core2 Duo CPU (2.67 GHz) and 2 Giga byte memory. The algorithms are validated and implemented under the environment of Matlab R2011a. Each node is a particle, the position of each node is composed of binary ordered pairs of keywords that are used to constitute questions and answers, so the space dimension is two-dimensional.

\subsection{Experiment One-Average-Radius Changing Trend}

Set the minimum correlation threshold $\xi=0.5$, the minimum fitness threshold $\lambda=0.5$. Firstly, particle are processed initially, initial position of each node is randomly generated. In Figure 1 and Figure 2, as can be seen from the Abscissa, the number of iterations is both set to 15 . When particle swarm executes each round of iteration, the average radius change trend is shown in Figure 1 and Figure 2 on the above two mentioned datasets. 
Values from the vertical axis represent the average radius of particle swarm optimization of all the sub community when performing each round of iteration.

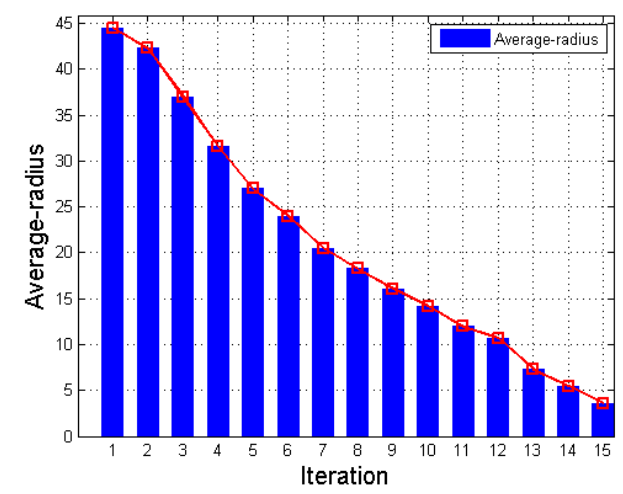

Figure 1. Average-Radius Changing Trend of Particle Swarm from DBLP

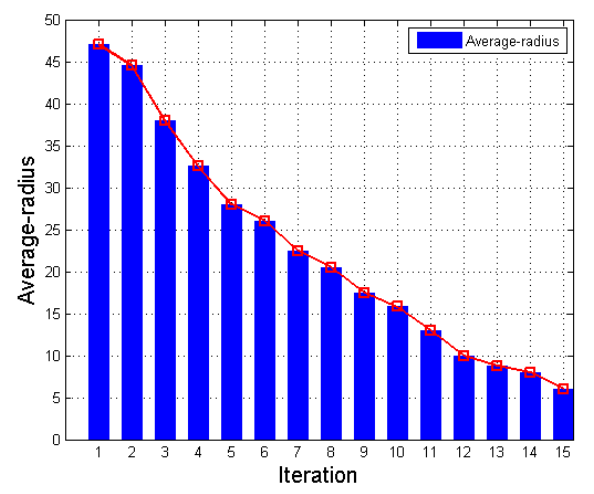

Figure 2. Average-Radius Changing Trend of Particle Swarm from Baidu Zhidao

From the above experiments, it can be found out that in the initial stage, on the experimental datasets from DBLP and Baidu Zhidao, the distribution structure of node particles are very loose, the connection between each particle is relatively sparse. Analyzing the change trend of particle swarm average radius of nodes in the sub community through the 15 times iteration, we can conclude that, with the continue of iteration step, the average radius of sub community is smaller and smaller, nodes with the same or similar content features gradually gathered together and lastly form a high cohesion, low coupling sub particle swarm community. This phenomenon shows that particle nodes with similar attributes and characteristics are gathered together in the process of continuous communication and interaction and eventually form a stable sub community.

\subsection{Experiment Two-Comparative Experiments}

\section{(1) Metrics}

The proposed algorithm aims to make community topic more refinement and precise for users, so we adopt the following metrics, which are commonly used in information retrieval and Web mining to evaluate the performance of all models. These metrics are precision rate and recall rate, which are all based on contingency matrix. The values in the contingency matrix represent the document number that meet the requirements, the details are shown in Table 3: 
Table 3. Contingency Matrix of Community Topic Refinement Strategy

\begin{tabular}{|c|c|c|}
\hline & $\begin{array}{c}\text { In the marked sub } \\
\text { topic community }\end{array}$ & $\begin{array}{c}\text { Not in the marked sub } \\
\text { topic community }\end{array}$ \\
\hline $\begin{array}{c}\text { In the topic search } \\
\text { results }\end{array}$ & $\mathrm{a}$ & $\mathrm{b}$ \\
\hline $\begin{array}{c}\text { Not in the topic search } \\
\text { results }\end{array}$ & $\mathrm{c}$ & $\mathrm{d}$ \\
\hline
\end{tabular}

Based on the above contingency matrix, precision rate and recall rate are defined respectively as follows:

$$
\begin{gathered}
\text { Pr ecision }=\frac{a}{a+b} \quad((a+b)>0) \\
\left.\operatorname{Re} \text { call }=\frac{a}{a+c}((a+c)>)\right)
\end{gathered}
$$

Where, precision rate denotes the proportion that the documents which really belong to the sub topic community document in all the detected result document, and recall rate is the ratio that the detected result documents are in all the marked sub community.

(2) Baselines and comparison

In experiments, the following state-of-the-art methods are evaluated for comparison, including our model, PISA model [15] and CR2NDAS model [16]. Introductions about PLSA and CR2NDAS are as follows:

PLSA model: It is a probabilistic latent semantic analysis (PLSA) model proposed by Hofmann in 2001, which makes words and documents map to the latent semantic space by dimension reduction, and remove some noise of the original vector space, semantic structure gradually presents, so as to find out the true meaning of the word in documents and queries, so it is latent semantic. Semantic structure gradually unfolds and the true meaning of words in documents and queries is found out, that is underlying semantics. But there is the problem of over fitting in PLSA model, that is to say with the increase in the number of documents sets, the number of model parameters will also increase accordingly.

CR2NDAS model: It is a new dynamic properties similarity calculation method between new nodes and also a community recommendation method based on dynamic attributes similarity of nodes. From the point of the dynamic perspective, considering the variation characteristics of social networks, through analyzing the social network dynamic evolution and the dynamic behavior information of individual network and links between individuals, dynamic attribute similarity between an individual and other individual behavior is calculated, the current communities where similar individuals are in are recommended to this individual.

\section{(3) Comparative experimental results analysis}

The comparative experiments adopt precision rate and recall rate as the evaluation index and use 10-fold cross-validation to test the accuracy of the models.10-fold crossvalidation algorithm is a commonly used testing methods. The data set is divided into ten parts which is in turn the nine parts as training data and the rest one as test data for test. Each test will get the corresponding accuracy or error rate. The average value of the 
correct rate or the error rate of 10 times is used to estimate the accuracy of the algorithm.The comparative experimental results on DBLP dataset and Baidu Zhidao dataset are as follws respectively.

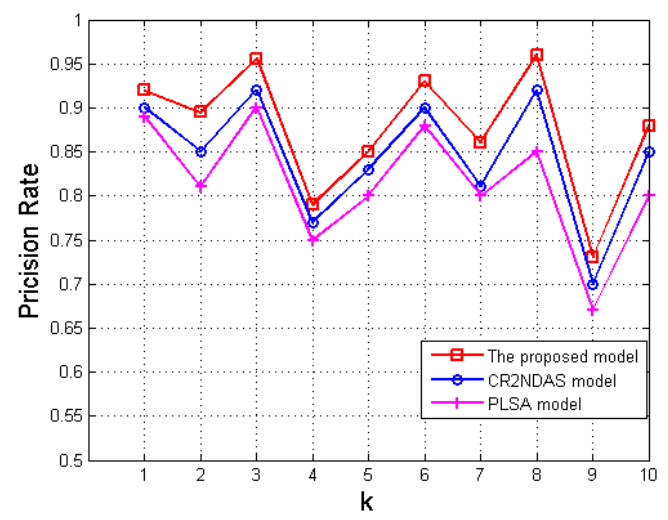

(a) Comparison On Precision Rate

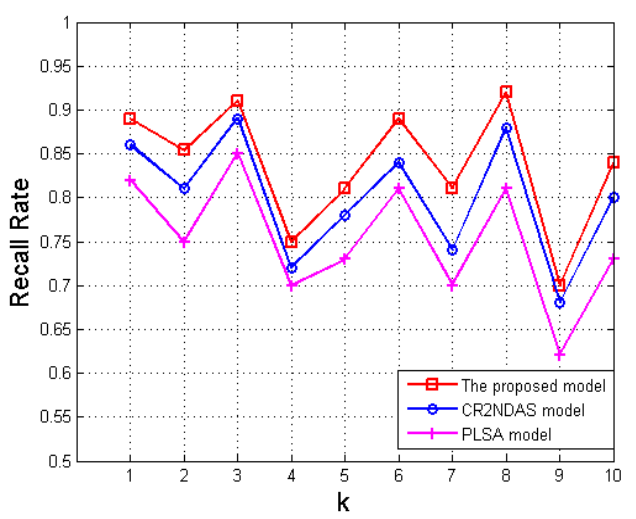

(B) Comparison On Recall Rate

Figure 3. Comparative Experimental Results Analysis on DBLP Data Set

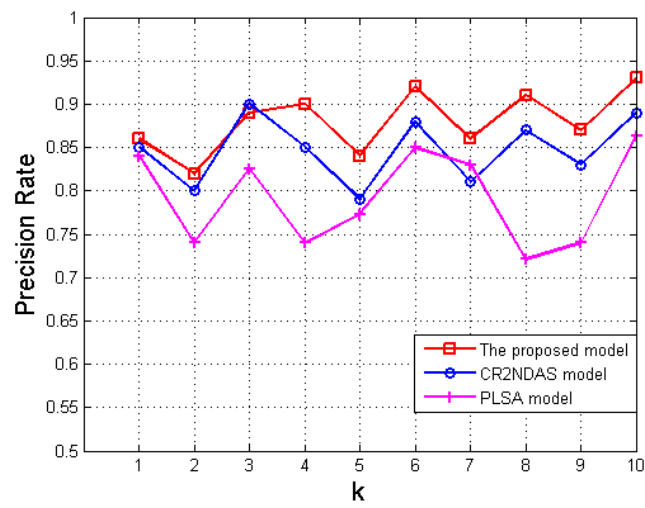

(A) Comparison on Precision Rate

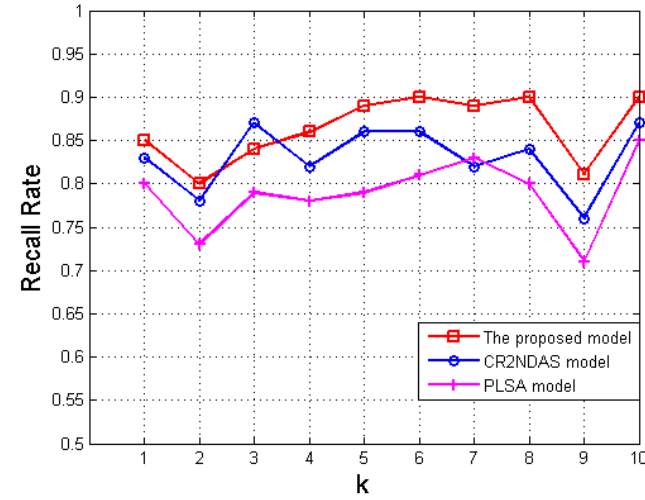

(B) Comparison on Recall Rate

\section{Figure 4. Comparative Experimental Results Analysis on Baidu Zhidao Dataset}

The experimental results are shown in Figure 3 and Figure 4. From Figure 3, it can be observed that among the baseline methods, our approach generally achieves the best precision rate and recall rate in every-fold cross-validation on DBLP dataset. From Figure 4 , it can be found out that, besides the third cross-validation, our proposed model outperforms CR2NDAS model and PLSA model about precision rate and recall rate on Baidu Zhidao dataset. About comparison between CR2NDAS model and PLSA model, in the 7th cross-validation, the precision rate and recall rate of CR2NDAS model are lower than PLSA model, in additon, in the rest nine cross-validations, CR2NDAS model are all superior to PLSA model. So we conclude that the average precision rate and average recall rate of the proposed method both outperform CR2NDAS model, and CR2NDAS model outperforms PLSA model. In a word, the improvements of our approach are significant, which justifies that our proposed strategy make the community topic be more refinement by using the improved PSO algorithm. 


\section{Conclusion and Future Work}

With the growing posts under the online community, the community topic refinement algorithm is studied based on the improved PSO algorithm. Contents of community nodes are performed dominant mining and in-depth analysis, we not only consider the explicit structure between the nodes, and emphatically analyzed the attributes and characteristics of the nodes, do more fine division to the sub topics of the community. Experimental results show that the proposed community topic refinement strategy based on the improved PSO algorithm outperforms the baselines CR2NDAS model and PLSA model and has a very good application prospect. However, in our current work, communities are only treated to be independent disjoint. In future work, we aim to make the proposed algorithm be applied to the overlapping community for topic refinement.

\section{Acknowledgements}

This work was supported by the key project of Anhui Province Colleges and Universities Natural Science Foundation of China (No.KJ2014A250), the project of outstanding young talents supported by Suzhou University of China (No.2014XQNRL009, No.2014XQNRL006, No.2014XQNRL008) and the project on the leader of information management and information system profession in Suzhou University (No.2014XJZY44).

\section{References}

[1] B. Lars, H. Dan and K. Jon, "Group formation in large social networks: Membership, growth, and evolution", Proceedings of the 12th ACM SIGKDD International Conference on Knowledge Discovery and Data Mining, New York, USA, (2006), pp. 44-54.

[2] A. Mislove, M. Marcon and K.P. Gummadi, "Measurement and analysis of online social networks", Proceedings of the 7th ACM SIGCOMM Conference on Internet Measurement, New York, USA, (2007), pp. 29-42.

[3] T.Y. Berger-Wolf and J. Saia, "A Framework for Analysis of Dynamic Social Networks", Proceedings of the 12th ACM SIGKDD International Conference on Knowledge Discovery and Data Mining, Philadelphia, USA, (2006), pp. 523-528;

[4] M.J. Newman, "Detecting community structure in networks", European Physical Journal B: Condensed Matter and Complex Systems, vol. 2, (2004), pp. 321-320.

[5] B. Saha and L. Getoor, "Group proximity measure for recommending groups in online social networks", "Proceedings of the 13th ACM SIGKDD International Conference on Knowledge Discovery and Data Mining", New York, USA, (2008), pp. 56-65.

[6] M.S. Handcock, A.E. Raftery and J.M. Tantrum, "Model-based clustering for social networks", Journal of the Royal Statistical Society: Series A, vol. 2, (2007), pp. 301-354.

[7] V. D. Blondel, J.L. Guillaume and R. Lambiotte, "Fast unfolding of communities in large networks", J. of Statistical Mechanics: Theory and Experiment, vol. 10, (2008), pp. 1-12.

[8] A. Tang and E. Viennet, "Community detection based on structural and attribute similarities", The Sixth International Conference on Digital Society, Valencia, Spain, (2012), pp. 7-12;

[9] T. Dinh, Y. Xuan and M.T. Thai, "Towards social-aware routing in dynamic communication networks", The 28th IEEE International Performance Computing and Communications Conference, Arizona, USA, (2009), pp. 161-168;

[10] C. Tantipathananandh and T.Y. Berger-Wolf, "Finding communities in dynamic social network", The 11th IEEE International Conference on Data Mining, Vancouver,Canada, (2011), pp. 1236-1241;

[11] N.P. Nguyen, T.N. Dinh and Y. Xuan, "Adaptive algorithms for detecting community structure in dynamic social networks", The 30th IEEE International Conference on Computer Communications, Shanghai, China, (2011), pp. 2282-2290;

[12] X.R. Wang and A. McCallum, "Topics over time: A non-Markov continuous-time model of topical trends", Proceedings of the 12th ACM International Conference on Knowledge Discovery and Data Mining, Philadelphia, USA, (2006), pp. 424-433.

[13] J. Kennedy and R.C. Eberhart, "Particle swarm optimization. Proceedings of IEEE International Conference on Neural Networks", Perth, WA, (1995), pp. 1942-1948.

[14] S. Solomon, P. Thulasiraman and R. Thulasiram, "Collaborative multi-swarm PSO for task matching using graphics processing units", Proceedings of the 13th Annual Conference on Genetic and Evolutionary Computation, Dublin, Ireland, Jul(2011), pp. 1563-1570. 
[15] T. Hofmann, "Probabilistic latent semantic indexing", Proceedings of the 22nd annual international ACM SIGIR conference on Research and development in information retrieval, California,USA, (1999), pp. 50-57.

[16] Q. Chen, H. Li and N.F. Xiao, "Community recommendation algorithm based on dynamic attributes similarity of nodes in social networks J. Computer Applications", vol. 5, (2010), pp. 1269-1271.
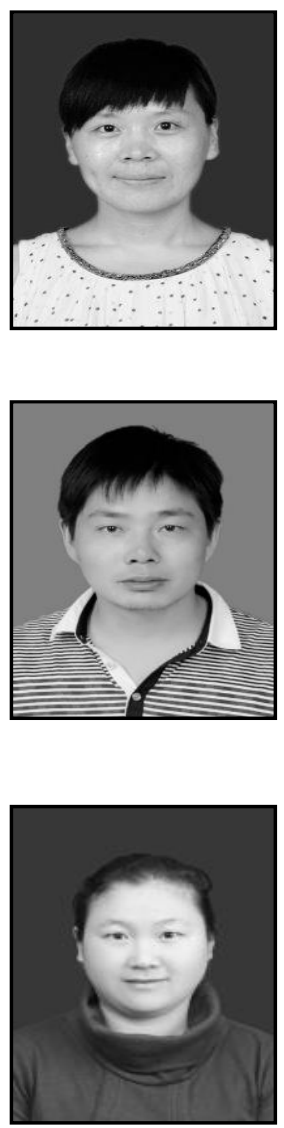

\section{Authors}

Lin Cui, She is studying for doctoral degree in Nanjing Universityof Aeronautics and Astronautics of China and she is also an researcher at Intelligent Information Processing Lab, Suzhou University, China. Her research interests include Web text mining and Social network.

Caiyin Wang, He received the master degree in computer science and technology from Hefei University of Technology, China in 2010. Currently, he is a researcher at Intelligent Information Processing Lab, Suzhou University, China. His research interests include Social Network and Semantic P2P.

Xiaoyin Wu, She received the master degree in computer science and technology from Hefei University of Technology, China. she is also a researcher at Intelligent Information Processing Lab Suzhou University, China. Her research interests include Internet of thing and Social Network. 
International Journal of Future Generation Communication and Networking Vol. 9, No. 1 (2016) 